\title{
A simple test for periodic signals in red noise
}

\begin{abstract}
S. Vaughan
X-Ray and Observational Astronomy Group, University of Leicester, Leicester, LE1 7RH, UK

e-mail: sav2@star.le.ac.uk

Received 11 June 2004 / Accepted 12 October 2004

Abstract. We demonstrate a simple method for testing the significance of peaks in the periodogram of red noise data. The procedure was designed to test for spurious periodicities in X-ray light curves of active galaxies, but can be used quite generally to test for periodic components against a background noise spectrum assumed to have a power law shape. The method provides a simple and fast test of the significance of candidate periodic signals in short, well-sampled time series such as those obtained from XMM-Newton observations of Seyfert galaxies, without the need for Monte Carlo simulations. A full account is made of the number of trials and the uncertainties inherent to the model fitting. Ignoring these subtle effects can lead to substantially overestimated significances. These difficulties motivate us to demand high standards of detection (minimum $>99.9$ per cent confidence) for periodicities in sources that normally show red noise spectra. The method also provides a simple means to estimate the power spectral index, which may be an interesting parameter itself, regardless of the presence/absence of periodicities.
\end{abstract}

Key words. methods: data analysis - methods: statistical - X-rays: general - X-rays: galaxies

\section{Introduction}

Many astrophysical sources show erratic, aperiodic brightness fluctuations with steep power spectra. This type of variability is known as red noise. By "noise" we mean to say that the intrinsic variations in the source brightness are random (this has nothing to do with measurement errors, also called noise). Examples include the X-ray variability of X-ray binaries (XRBs; e.g. van der Klis 1995) and Seyfert galaxies (e.g. Lawrence et al. 1987; Markowitz et al. 2003). The power spectrum of these variations, which describes the dependence of the variability amplitude on temporal frequency, is often reasonably approximated as a simple power law (over at least a decade of frequency). This featureless continuum spectrum does not offer any characteristic frequencies that could be used as diagnostics.

XRBs often show quasi-periodic oscillations (QPOs) that show-up as peaks in the power spectrum over the continuum noise spectrum. These can be thought of as half-way between strictly periodic variations (all power concentrated at one frequency) and broad-band noise (power spread over a very broad range of frequencies). A combination of periodic oscillations with similar frequencies, or a single oscillation that is perturbed in frequency, amplitude or phase can produce a QPO. QPOs are one of the most powerful diagnostics of XRB physics (see e.g. van der Klis 1995; $M^{\mathrm{c} C l i n t o c k} \&$ Remillard 2004). The detection of periodic or quasi-periodic variations from a Seyfert galaxy would a be a key observational discovery, and could lead to a breakthrough in our understanding if the characteristic (peak) frequency could be identified with some physically meaningful frequency. For example, if we assume a $1 / M_{\mathrm{BH}}$ scaling of frequencies we might expect to see analogues of the high frequency QPOs of XRBs in the range $f_{\mathrm{QPO}} \sim 3 \times 10^{-3}\left(M_{\mathrm{BH}} / 10^{6} M_{\odot}\right)^{-1} \mathrm{~Hz}$ (Abramowicz et al. 2004).

However, claims of periodic variations and QPOs in the $\mathrm{X}$-ray emission of Seyfert galaxies have a chequered history, with no single example withstanding the test of repeated analyses and observations (see discussion in Benlloch et al. 2001). The confusion arises partly due to the lack of a standard technique to assess the significance of a periodicity claim against a background assumption of random, red noise variability. Indeed, as Press (1978) and others have remarked, there is a tendency for the eye to identify spurious, low frequency periods in random time series. Tests for the presence of periodic variations against a background of white (flat spectrum) noise are well established, from Schuster (1898) and Fisher (1929), these are reviewed in Sect. 6.1.4 of Priestley (1981), and discussed in an astrophysical context by Leahy et al. (1983) and van der Klis (1989). But without modification these methods cannot be used to test against red noise variations. Timmer \& König (1995) and Benlloch et al. (2001) have proposed Monte Carlo testing methods applicable to red noise but the relatively high computational demands of these methods may be enough to deter some potential users. Israel \& Stella (1996) proposed a method that does not require Monte Carlo simulations but is not optimised for short observations of power law continuum spectra.

This paper puts forward a simple test that can be used to test the significance of candidate periodicities superposed on a red noise spectrum which has an approximately power law shape. The price of simplicity, in this case, is that the test is 
only strictly valid when the underlying continuum spectrum is a power law. The basic steps of the method are: $(i)$ measure the periodogram; (ii) estimate the red noise continuum spectrum; and (iii) estimate the significance of any peaks above the continuum. The stages of the method are explained in detail in the following sections. Section 2 gives a brief introduction to the statistical properties of the periodogram. Section 3 discusses a simple method for estimating the parameters of a power lawlike spectrum and Sect. 4 discusses how to estimate the significance of a peak above the continuum. Section 5 then demonstrates the veracity of the method using Monte Carlo simulations and Sect. 6 reviews some important caveats that must be considered when using this (and other) periodsearching methods. Finally, Sect. 7 gives a brief review of the method in the context of observations of active galaxies. The appendix discusses a more generally applicable method of periodogram fitting (that makes no assumption on the form of the underlying spectrum).

\section{The periodogram}

Given an evenly sampled time series $x_{k}$ of $K$ points sampled at intervals $\Delta T$ we can measure its periodogram (Schuster 1898), which is simply the modulus-squared of the discrete Fourier transform, $X\left(f_{j}\right)$, at each of the $n=K / 2$ Fourier frequencies:

$$
I\left(f_{j}\right)=\frac{2 \Delta T}{\langle x\rangle^{2} N}\left|X_{j}\right|^{2}
$$

The normalisation is chosen such that the units of the periodogram are (rms/mean) ${ }^{2} \mathrm{~Hz}^{-1}$ (where rms/mean is dimensionless) and summing the periodogram over positive frequencies gives the sample variance in fractional units. The periodogram is evaluated at the Fourier frequencies $f_{j}=j / K \Delta T$ with $j=1,2, \ldots, K / 2$. The original purpose of the periodogram was as a tool for identifying "hidden periodicities" in time series. However, the periodogram of a noise process, if measured from a single time series, shows a great deal of scatter around the underlying power spectrum. Specifically, the periodogram at a given frequency, $I\left(f_{j}\right)$, is scattered around the true power spectrum, $\mathcal{P}\left(f_{j}\right)$, following a $\chi^{2}$ distribution with two degrees of freedom:

$I\left(f_{j}\right)=\mathcal{P}\left(f_{j}\right) \chi_{2}^{2} / 2$,

where $\chi_{2}^{2}$ is a random variable distributed as $\chi^{2}$ with two degrees of freedom, i.e. an exponential probability density with a mean and variance of two and four, respectively:

$p_{\chi^{2}}(x)=\mathrm{e}^{-x / 2} / 2$.

The periodogram is distributed in this way because the real and imaginary parts of the DFT are normally distributed for a stochastic process ${ }^{1}$, and the sum of two squared normally distributed variables is a $\chi_{2}^{2}$-distributed variable (Jenkins \& Watts 1968; Priestley 1981; Chatfield 1989; Bloomfield 2000). See

\footnotetext{
${ }^{1}$ The DFT at the Nyquist frequency is always real when $N$ is even so the periodogram at this frequency is distributed as $\chi_{1}^{2}$, i.e. with one degree of freedom.
}

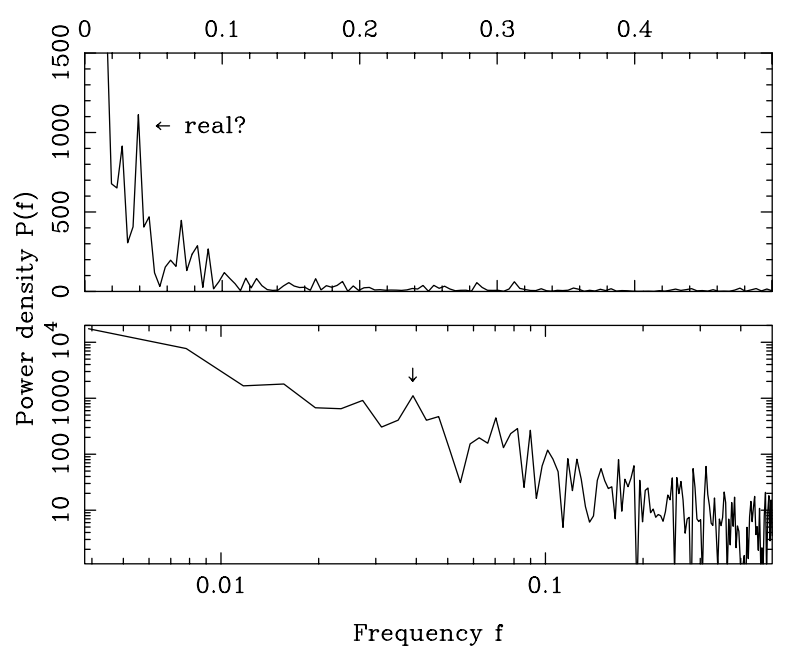

Fig. 1. Periodogram of a short $(K=256)$ time series containing red noise. The upper panel shows the periodogram using linear axes, the lower panel shows the same data on logarithmic axes. The periodogram shows a red noise spectrum rising at lower frequencies. But the periodogram also shows a peak at $f=4 \times 10^{-2}$. Is this due to a real harmonic (periodic) variation or an artifact of the fluctuating noise spectrum?

also Scargle (1982), Leahy et al. (1983), Wall \& Jenkins (2003) and Groth (1975) for further discussion of this point.

If the spectrum is flat ("white noise") and its power level known a priori then we can simply make use of the known probability distribution (Eqs. (2) and (3)) to estimate the likelihood that a given periodogram ordinate exceeds some threshold. If the power level is not known there is an added uncertainty, but nevertheless an exact test does exist (Fisher's $g$ statistic: Fisher 1929; Sect. 6.1 .4 of Priestley 1981) to estimate the likelihood that the highest peak in the periodogram was caused by a random fluctuation in the noise spectrum (see also Koen 1990).

For the more general case of non-white noise there is no such exact test. When examining the periodogram of red noise data such as from Seyfert galaxies, we need to be careful not to identify spurious peaks. Even in the "null" case (i.e. no periodic component) peaks may occur in the periodogram due to sampling fluctuations. In particular the eye may be drawn to low frequency peaks because, in red noise data, there is much more power and more scatter in the periodogram at low frequencies. Given a large amount of data we can average the periodogram in one of the standard ways (see e.g. van der Klis 1989; Papadakis \& Lawrence 1993a), fit the continuum using a standard $\chi^{2}$-minimisation tool (e.g. Bevington \& Robinson 1992; Press et al. 1996) and test of the presence of addition features. This is the standard procedure for analysing XRB data. If we have a very limited amount of data, such that we cannot afford to average the periodogram, we are faced with a more difficult situation. Figure 1 gives an example like this. The periodogram of a short time series, containing red noise (generated using the method of Timmer \& König 1995), shows a large peak at $f=4 \times 10^{-2}$. Could this be due to a real periodic variation present in the data or is it just a fluctuation in the red noise spectrum? 


\section{Fitting the periodogram}

\subsection{Least squares (LS) fit to log-periodogram}

If the underlying power spectrum is suspected to be a power law then the parameters of interest are its slope, $\alpha$, and normalisation $N$. One of the simplest methods to estimate these parameters from the raw (unbinned) periodogram is to fit it with a model of the form $\mathcal{P}(f)=N f^{-\alpha}$ using the method of least squares (LS). The problem with this is that the periodogram is distributed around the true underlying spectrum in a non-Gaussian fashion and, more seriously, the distribution depends on the spectrum itself (Eq. (2)).

To simplify the problem we can fit the logarithm of the periodogram, as discussed in some detail by Geweke \& Porter-Hudak (1983; see also Papadakis \& Lawrence 1993a). The scatter in the periodogram scales with the spectrum itself; the scatter is multiplicative in linear-space. This means the scatter is additive in log-space:

$\log \left[I\left(f_{j}\right)\right]=\log \left[\mathcal{P}\left(f_{j}\right)\right]+\log \left[\chi_{2}^{2} / 2\right]$

and therefore identical at each frequency (the data are homoskedastic). Working with the logarithm of the periodogram has two further advantages. The first is that if the power spectrum is a power law, the natural way to plot the data is in log space. The power law becomes a linear function: $\log [\mathcal{P}(f)]=\log [N]-\alpha \log [f]$. The second advantage is that the distribution of periodogram ordinates becomes less skewed. This reduces the effect of "outliers" on the fitting.

We must be careful fitting the logarithm. The expectation value of the logarithm of the periodogram is not the expectation value of the logarithm of the spectrum. However, the bias is a constant (due to the shape of the $\chi_{2}^{2}$-distribution in log-space) that can be removed trivially:

$\left\langle\log \left[I\left(f_{j}\right)\right]\right\rangle=\left\langle\log \left[\mathcal{P}\left(f_{j}\right)\right]\right\rangle+\left\langle\log \left[\chi_{2}^{2} / 2\right]\right\rangle$.

Using Eq. (26.4.36) of Abramowitz \& Stegun (1964) we have $\left\langle\log \left[\chi_{2}^{2} / 2\right]\right\rangle=-0.57721466 \ldots / \ln [10]$. (The number in the numerator is Euler's constant.) Therefore

$\left\langle\log \left[\mathcal{P}\left(f_{j}\right)\right]\right\rangle=\left\langle\log \left[I\left(f_{j}\right)\right]\right\rangle+0.25068 \ldots$

The logarithm of the periodogram ordinate, with the bias removed (i.e. the 0.25068 added $^{2}$ ), is thus an unbiased estimator of the logarithm of the spectrum, and is distributed independently and identically (about the underlying spectrum) at each frequency. (The raw periodogram points are not identically distributed in linear space since the scatter depends on the spectrum, which is a function of frequency.) Thus we can use a LS fitting procedure to get a reasonable estimate of the power spectral slope $\alpha$ and normalisation $N$ by fitting a linear function $y=m x+c$ to the plot of $\log \left[I\left(f_{j}\right)\right]$ versus $\log \left[f_{j}\right]$. The slope of the linear fit gives $\hat{\alpha}=-m$ and the $y$-intercept gives $\log (\hat{N})=c+0.25068$. These give our estimate for the continuum: $\hat{\mathcal{P}}_{j}=\hat{N} f_{j}^{-\hat{\alpha}}$, or equivalently $\log \left[\hat{\mathcal{P}}_{j}\right]=\log [\hat{N}]-\hat{\alpha} \log \left[f_{j}\right]$.

It is important to note that the datum at the Nyquist frequency $(j=n)$ should be ignored in the LS fitting. This is

\footnotetext{
2 This is a more precise approximation to $\left\langle\log \left[\chi_{2}^{2} / 2\right]\right\rangle$ than used by Papadakis \& Lawrence (1993a).
}

because, as mentioned previously, the distribution of the periodogram ordinate at this frequency is not identical to that at other frequencies (it follows a $\chi_{1}^{2}$ distribution). This minor detail means that the LS fit should be performed on the $n^{\prime}=n-1$ lowest frequencies that are identically distributed (in log-space).

A drawback of fitting the periodogram, rather than the binned or averaged periodogram, is that it does not provide a in-built goodness-of-fit test. By binning the periodogram (van der Klis 1989; Papadakis \& Lawrence 1993a) we can obtain Gaussian errors on each ordinate to be used in a $\chi^{2}$-test. We do not have Gaussian error bars for the unbinned logperiodogram. But, since we know the expected distribution of the periodogram ordinates about the true spectrum we can compare this to the distribution of residuals from the fitted data using a Kolmogorov-Smirnov test (Press et al. 1996). Specifically, we can compare the data/model ratio (in linear space) given by $\hat{\gamma}_{j}=2 I_{j} / \hat{\mathcal{P}}_{j}$ with the theoretical $\chi_{2}^{2}$ distribution, if the model is reasonable $\hat{\gamma}_{j}$ should be consistent with the $\chi_{2}^{2}$ distribution. Furthermore, the KS test is most sensitive around the median value, and less sensitive at the tails of the distribution, which means that even in the presence of a real periodic signals (i.e. a few outlying powers) the test should give a good idea of the overall quality of the continuum fit.

\subsection{Uncertainties on the parameters}

The uncertainties on the slope and normalisation estimates from the LS method can be derived using the standard theory of linear regression (e.g. Bevington \& Robinson 1992; Press et al. 1996). The error on the slope (index) and intercept (log normalisation) are:

$\operatorname{err}^{2}[\hat{\alpha}]=\frac{n^{\prime} \sigma^{2}}{\Delta}$

and

$\operatorname{err}^{2}[\log (\hat{N})]=\frac{\sigma^{2} \sum_{j=1}^{n^{\prime}} a_{j}^{2}}{\Delta}$

where $a_{j}=\log \left[f_{j}\right]$ and

$\Delta=n^{\prime} \sum_{j=1}^{n^{\prime}} a_{j}^{2}-\left(\sum_{j=1}^{n^{\prime}} a_{j}\right)^{2}$

and also $\sigma^{2}=\pi^{2} / 6(\ln [10])^{2}$ is the variance of the logperiodogram ordinates about the true spectrum (Geweke \& Porter-Hudak 1983). The covariance of the two parameter estimates is given by:

$\operatorname{cov}[\hat{\alpha}, \log (\hat{N})]=\frac{\sigma^{2} \sum_{j=1}^{n^{\prime}} a_{j}}{\Delta}$.

Here $n^{\prime}$ is the number of frequencies used in the fitting. Normally $n^{\prime}=n-1$ since only the Nyquist frequency is ignored (because the periodogram at the Nyquist frequency does not follow the same distribution as at the other frequencies).

The accuracy of these equations was tested using a Monte Carlo simulations. An ensemble of random time series, each 

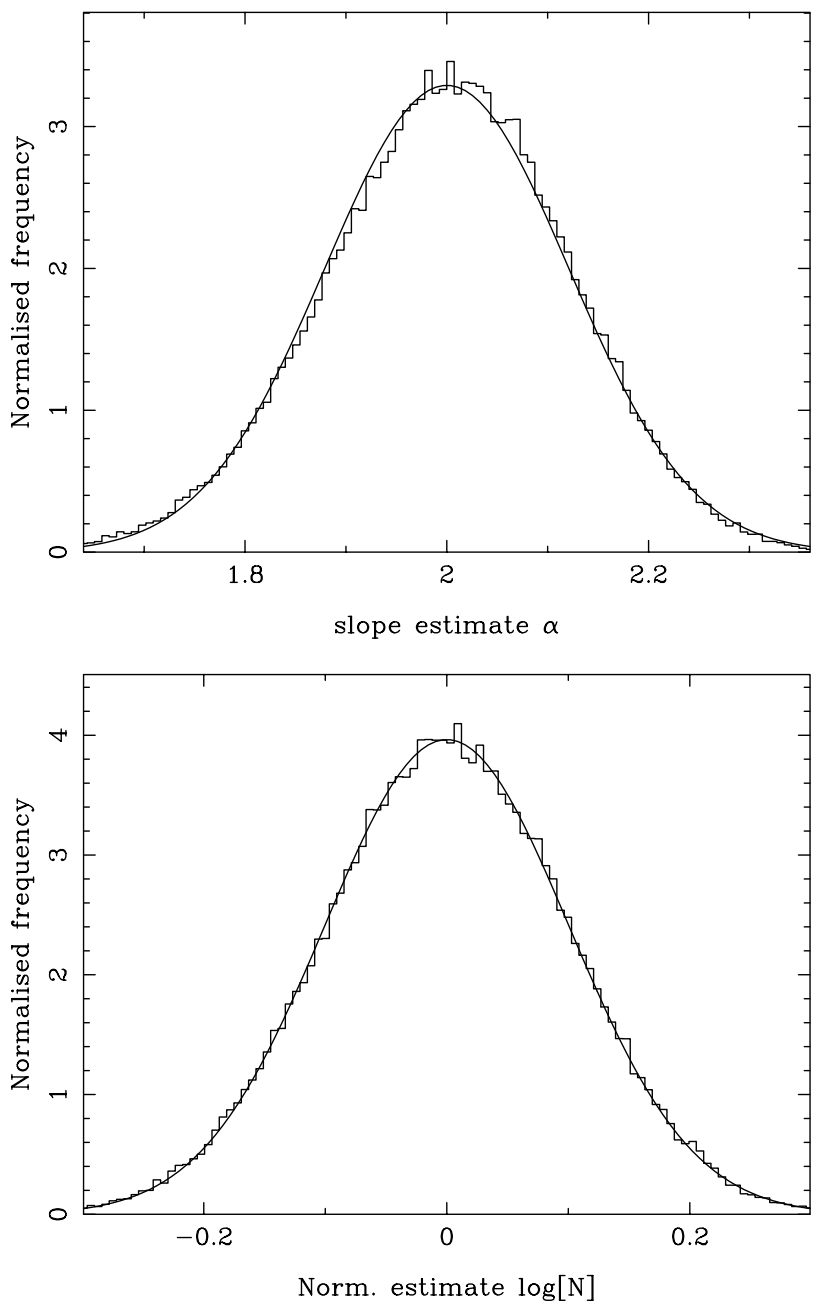

Fig. 2. Distribution of the slope and normalisation estimators derived from $10^{5}$ Monte Carlo simulations of $K=256$ point time series (histogram). The "true" spectral parameters were $\alpha=2$ and $\log [N]=$ 0 . The predictions of Gaussian uncertainties, with widths given by Eqs. (7) and (8), are shown with the smooth curves.

of length $K$, was generated (using the method of Timmer \& König 1995). For each series the power spectral slope and normalisation were estimated using the LS method discussed above. Figure 2 shows the distribution of estimates for $10^{5}$ realisations of time series generated by a process with an $\alpha=$ $2, N=1$ spectrum. With only $n=127$ periodogram points (i.e. $K=256$ ) the distribution of the estimates is reasonably close to Gaussian. (The distribution of $\hat{N}$ is log-normal because the estimated quantity $\log [\hat{N}]$ is normally distributed in the LS fitting.) These two parameters are covariant in the fit; a low estimate of the slope tends to be correlated with a high estimate of the normalisation. Figure 3 illustrates the covariance between the two estimated parameters. The shape of these distributions is independent of the spectral slope, this was confirmed using Monte Carlo simulations of spectra with slopes in the range $\alpha=0-3$.

The uncertainties on $\hat{\alpha}$ and $\log [\hat{N}]$, and their covariance, were estimated for different length series by the same Monte Carlo method as discussed above. These Monte Carlo

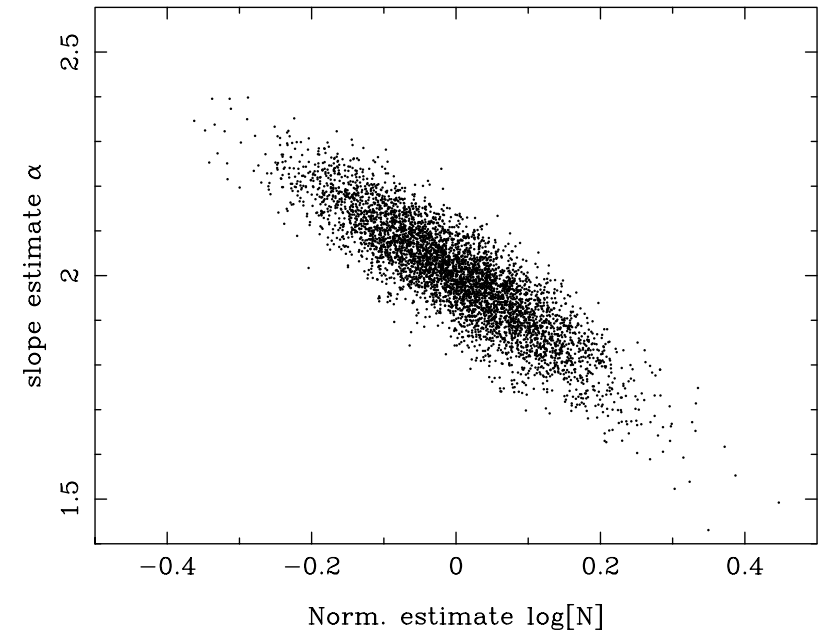

Fig. 3. Demonstration of the covariance in the estimates of slope and normalisation from from LS fitting to the logarithm of the periodogram. The plot shows the results of fitting 5000 Monte Carlo simulations of an $\alpha=2, N=1$ spectrum.

uncertainties compare well with the theoretically expected uncertainties for the LS fitting method as discussed above (Fig. 4).

\subsection{Uncertainty on the model}

The expected uncertainties and covariance of the two model parameters can be combined to give an estimate of the uncertainty of the logarithm of the model, $\log \left[\hat{\mathcal{P}}_{j}\right]$, at a frequency $f_{j}$, using the standard error propagation formula.

$$
\begin{aligned}
\operatorname{err}^{2}\left[\log \left\{\hat{\mathcal{P}}\left(f_{j}\right)\right\}\right]= & \operatorname{err}^{2}[\hat{\alpha}] \times\left(\log \left[f_{j}\right]\right)^{2}+\operatorname{err}^{2}[\log (\hat{N})] \\
& -2 \operatorname{cov}[\alpha, \log (\hat{N})] \times\left(\log \left[f_{j}\right]\right) .
\end{aligned}
$$

The first term in the sum accounts for the uncertainty on the slope (Eq. (7)), the second accounts for the uncertainty in the normalisation (Eq. (8)) and the third accounts for their covariance (Eq. (10)). The uncertainty is frequency dependent. Even in log-space the model is more uncertain at lower frequencies, this simply reflects the fact that there are many more points at high $\log [f]$ than at $\operatorname{low} \log [f]$. But also note that the uncertainty on the logarithm of the model is independent of the model itself (slope and normalisation).

The distribution of the power in the model (in log-space), $\log \left[\mathcal{P}_{j}\right]$, is expected to be Gaussian with a width determined by the formula above. In linear-space the uncertainty on the model power, $\mathcal{P}_{j}$, is log-normally distributed. The probability density function (PDF) for the model power is therefore

$p_{\hat{\mathcal{P}}_{j}}(y)=\frac{1}{S_{j} y \sqrt{2 \pi}} \exp \left\{-\frac{\left(\ln [y]-M_{j}\right)^{2}}{2 S_{j}^{2}}\right\}$

where $M_{j}=\ln \left[\mathcal{P}_{j}\right]$ is the expected value of the power (in log-space) and $S_{j}$ is the rms width of the distribution of powers (also in log-space) as given by Eq. (11). Both of these are frequency dependent. Note that the log-normal distribution is conventionally defined in terms of the natural logarithm, whereas previously the results were given in terms of 


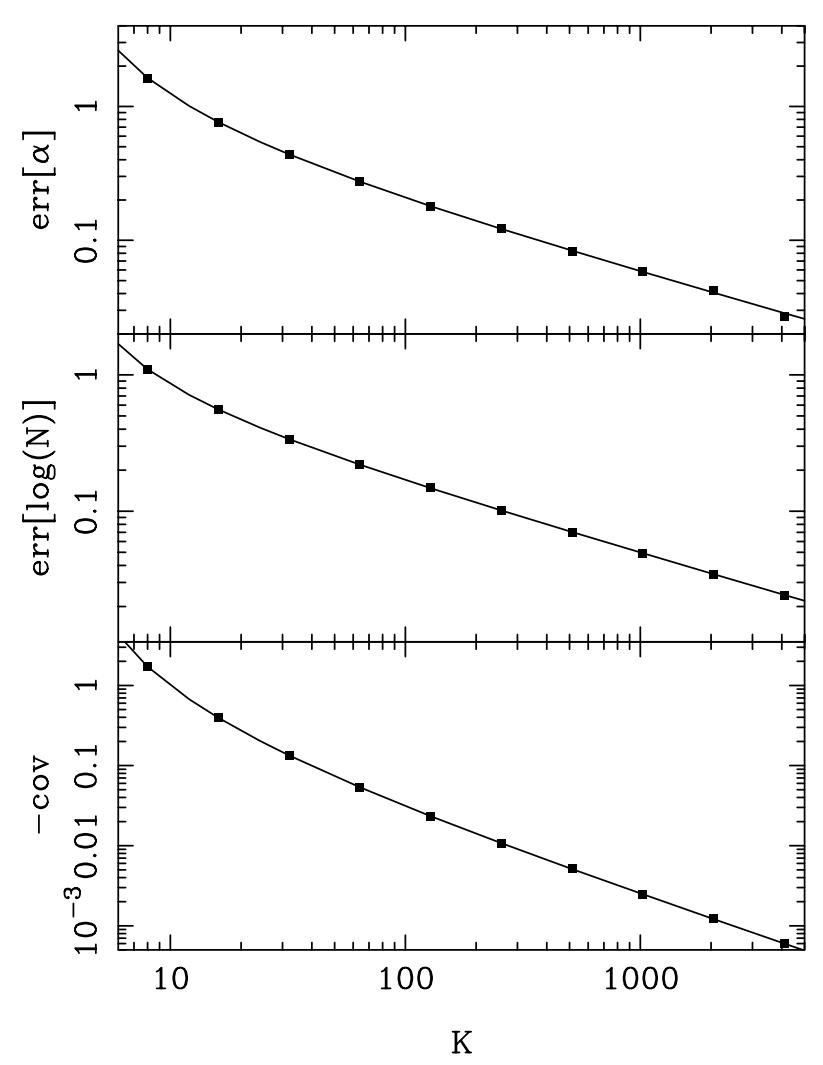

Fig. 4. Demonstration of the uncertainties from LS fitting to the logarithm of the periodogram. For each value of $K, 10^{5}$ time series were simulated (with an $\alpha=2, N=1$ spectrum). The periodogram of each time series was fitted (in log space) with a linear model. From the $10^{5}$ estimates of the two parameters (slope and normalisation) their rms and covariance were measured (solid squares). The solid lines mark the predictions of Eqs. (7), (8) and (10).

base 10 logarithm. The uncertainty on the model log-powers from Eq. (11) needs to be corrected:

$S_{j}=\operatorname{err}\left[\log \left\{\hat{\mathcal{P}}\left(f_{j}\right)\right\}\right] \times \ln [10]$.

Figure 5 shows the distribution of model powers (at two different frequencies) for $10^{5}$ Monte Carlo simulations of $K=$ 256 time series. Clearly the predicted log-normal distribution (with parameters $M_{j}$ and $S_{j}$ as given above) gives a good description of the real uncertainty in the model.

\subsection{Bias in the parameters}

Although in general the LS method does not yield the maximum likelihood solution for non-Gaussian data, for the specific case of a power law spectrum the parameters obtained from the log-periodogram regression, namely $\hat{\alpha}$ and $\log [\hat{N}]$, are unbiased. Figure 6 demonstrates this using Monte Carlo simulations. However, it should be noted that because the parameter $\log [\hat{N}]$ is normally distributed the parameter $\hat{N}$ will be log-normally distributed. Thus the mean value of $\hat{N}$ is not a good estimator of the true value (it will be biased upwards due to the long tail of the log-normal distribution).

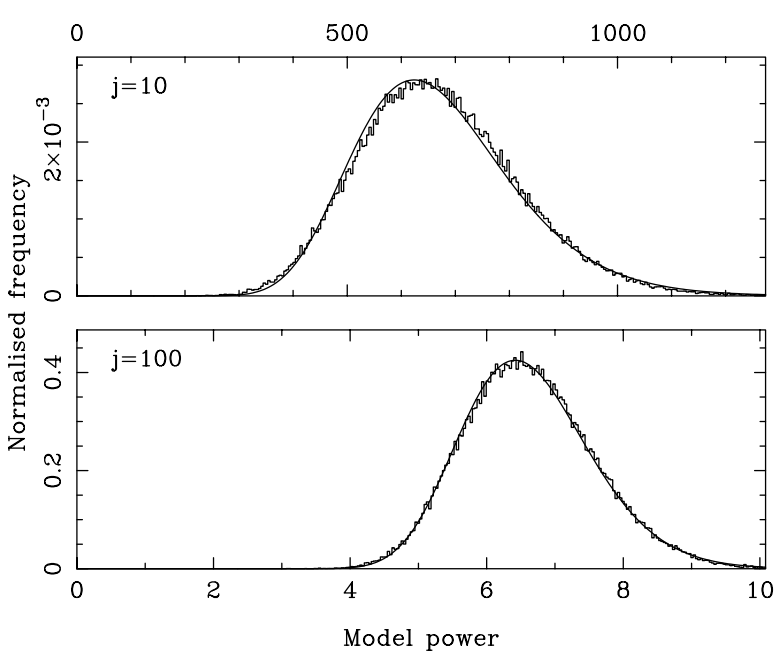

Fig. 5. Monte Carlo demonstration of the distribution of power in the model (power law) spectrum. Using an $\alpha=2, N=1$ spectrum, $10^{5}$ random time series of length $K=256$ were generated. For each one the periodogram was fitted as discussed in the text. The histograms show the distribution of the power in the resulting $10^{5}$ models at the $j=10$ and $j=100$ Fourier frequencies $\left(f_{j}=j / K \Delta T\right)$. The solid line shows the predicted log-normal distribution (Eq. (12)).

\subsection{Summary of LS fitting}

The following summarises the LS fitting method:

- Measure the periodogram of the time series.

- Ignore the Nyquist frequency point.

- Take the logarithm.

- Fit a straight line using the standard LS method.

- Test the goodness-of-fit using the KS test.

- Estimate uncertainties on the parameters and the model.

The uncertainties and covariance of the parameters can be computed using Eqs. (7), (8) and (10). These can then be used to calculate the uncertainty on the logarithm of model power $S_{j}$ (Eq. (13)).

\section{Confidence limits}

\subsection{The ideal case}

If we know the exact form of the spectrum we can divide this out of the periodogram. From Eq. (2) we can see that the ratio $\gamma_{j} \equiv 2 I\left(f_{j}\right) / \mathcal{P}\left(f_{j}\right)$ will be distributed like $\chi_{2}^{2}$. We can use our estimates $\hat{\alpha}$ and $\hat{N}$ to form the null hypothesis: the data were generated by a process with a spectrum $\hat{\mathcal{P}}\left(f_{j}\right)=\hat{N} f_{j}^{-\hat{\alpha}}$ and no periodic component. We can estimate the probability that a large peak will occur in the periodogram, assuming the model spectrum, by comparing $\hat{\gamma}_{j}$ to the $\chi_{2}^{2}$ PDF (Priestley 1981; Scargle 1982).

We can define a $(1-\epsilon) 100$ per cent confidence limit on $\hat{\gamma}$, call this $\gamma_{\epsilon}$, as the level for which, at a given frequency, the probability of obtaining a higher value by chance is $\operatorname{Pr}\{\hat{\gamma}>$ $\left.\gamma_{\epsilon}\right\}=\epsilon$ on the assumption that the null hypothesis is true. The chosen value of $\epsilon$ represents the "false alarm probability". 


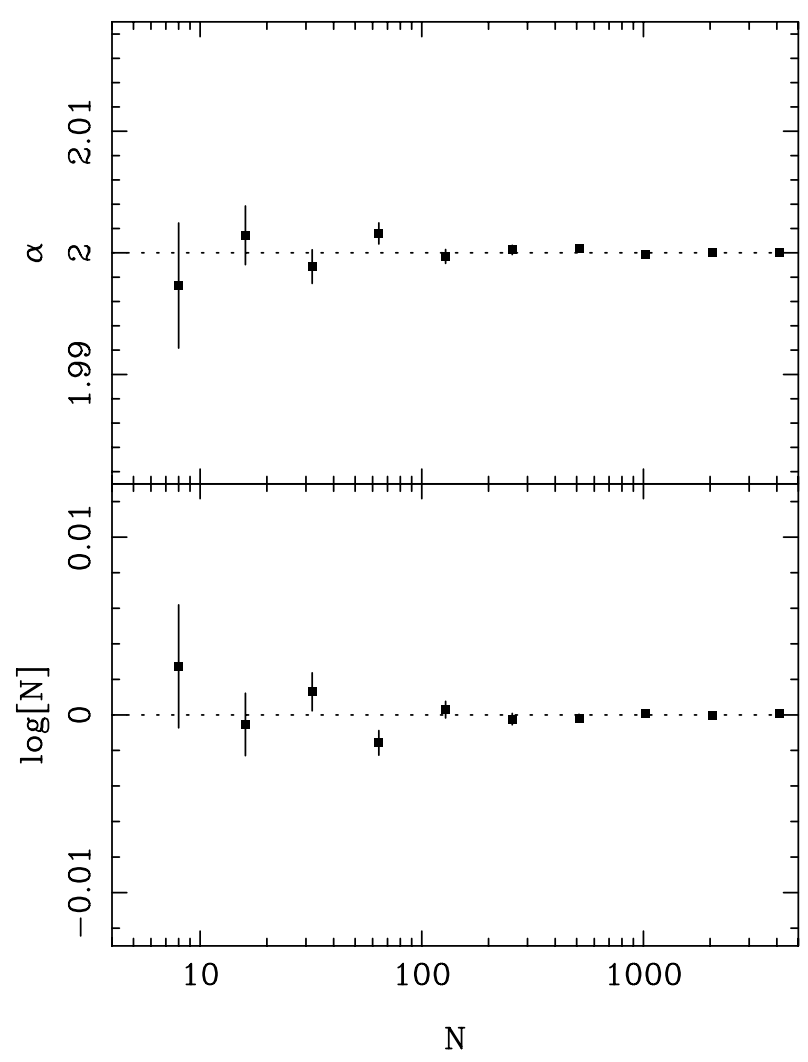

Fig. 6. Demonstration of bias in LS fitting of the logarithm of the periodogram. For each value of $K, 10^{5}$ time series were simulated (with an $\alpha=2, N=1$ spectrum) and the periodogram of each time series was fitted (in log space; see text) with a linear model. The parameters estimates were averaged over the $10^{5}$ realisations. The estimators $\hat{\alpha}$ and $\log [\hat{N}]$ are clearly unbiased.

The integral of the $\chi_{2}^{2}$ probability density gives the probability of a single sample exceeding a value of $\gamma_{\epsilon}$ by chance:

$\operatorname{Pr}\left\{\chi^{2}>\gamma_{\epsilon}\right\}=\frac{1}{2} \int_{\gamma_{\epsilon}}^{\infty} \mathrm{e}^{-x / 2} \mathrm{~d} x=\mathrm{e}^{-\gamma_{\epsilon} / 2}=\epsilon$.

For a given probability $\epsilon$, we can rewrite this:

$\gamma_{\epsilon}=-2 \ln [\epsilon]$.

For example, using $\epsilon=0.05$ (i.e. a 95 per cent significance test) we find $\gamma_{0.05}=5.99$. This means that if the null hypothesis is true the probability of the ratio $\hat{\gamma}_{j}$ being higher than 5.99 is only 0.05 . We can therefore define our 95 (and 99) per cent confidence limits on the log-periodogram as the model $\hat{\mathcal{P}}(f)=\hat{N} f^{-\hat{\alpha}}$ multiplied by the appropriate $\gamma_{\epsilon} / 2$. (In log-space we simply add the appropriate $\log \left[\gamma_{\epsilon} / 2\right]$ to the model.)

However, these confidence bounds correspond to a single trial test, they give the probability that a periodogram point at one particular frequency will exceed $\gamma_{\epsilon} \mathcal{P}_{j} / 2$. Usually there are $n^{\prime}=n-1$ independent trials since only the Nyquist frequency is ignored (leaving $n^{\prime}$ independently distributed periodogram points to be examined). We must account for the number of independent trials:

$\gamma_{\epsilon}=-2 \ln \left[1-\left(1-\epsilon_{n^{\prime}}\right)^{1 / n^{\prime}}\right]$.

This gives the value of $\hat{\gamma}$ that has a probability of being exceeded of $\epsilon_{n^{\prime}}$ in $n^{\prime}$ independent trials. In the limit of large $n^{\prime}$ and small $\epsilon$ this can be approximated as $\gamma_{\epsilon}=-2 \ln \left[\epsilon_{n^{\prime}} / n^{\prime}\right]$. Of course, if we knew which frequencies to test a priori then we could perform $<n^{\prime}$ independent tests and the significance levels could be adjusted accordingly.

\subsection{Accounting for model uncertainty}

The case outlined above is valid only when we know the true power spectrum exactly $\left(\hat{\mathcal{P}}_{j}=\mathcal{P}_{j}\right)$. In reality all we have is an estimated model $\hat{\mathcal{P}}_{j}$ (which will differ from the true spectrum) and its uncertainty. This extra uncertainty alters the probability distribution. The ratio $\hat{\gamma}_{j}=2 I_{j} / \hat{\mathcal{P}}_{j}$ is really the ratio of two random variables; the PDF of this would allow us to calculate the probability of observing a given value of $\hat{\gamma}_{j}$ taking full account of the uncertainty in the model fitting. As discussed above $2 I_{j}$ will follow a rescaled $\chi_{2}^{2}$ distribution about the true spectrum.

$p_{2 I_{j}}(x)=\frac{1}{2 \mathcal{P}_{j}} \mathrm{e}^{-x / 2 \mathcal{P}_{j}}$.

In the case of the LS fitting discussed in Sect. 3 the model $\hat{\mathcal{P}}_{j}$ has a log-normal distribution. The probability density of the power in the fitted model at frequency $f_{j}$ is therefore:

$p_{\hat{\mathcal{P}}_{j}}(y)=\frac{1}{S_{j} y \sqrt{2 \pi}} \exp \left\{-\frac{\left(\ln [y]-M_{j}\right)^{2}}{2 S_{j}^{2}}\right\}$

where $M_{j}=\ln \left[\mathcal{P}_{j}\right]$ and $S_{j}$ is the uncertainty on the logarithm of the model (Eq. (13)). The periodogram ordinate $I_{j}$ and the power $\hat{\mathcal{P}}_{j}$ in the best fitting model are only strictly independent if the frequency of interest, $f_{j}$, is excluded from the LS fit. Otherwise the fitted model, and hence the model power at this frequency, $\hat{\mathcal{P}}_{j}$, is influenced by $I_{j}$. Although the effect is small $(\sim 1 / n)$ it does have a substantial impact on the tail of the PDF. Thus, to obtain formally independent variables we must calculate $\hat{\mathcal{P}}_{j}$ (and its error) using the LS method after ignoring $I_{j}$ from the fit. In other words, we must ignore the candidate frequency when fitting the continuum model and then compare the measured periodogram ordinate at this frequency with the model derived from fitting all the other (independent) frequencies.

The PDF of the ratio $\hat{\gamma}_{j}$ can be obtained using the standard formula for the PDF of the ratio of two independent variables:

$p_{\gamma_{j}}(z)=\int_{-\infty}^{+\infty}|y| p_{2 I_{j}}(z y) p_{\mathcal{P}_{j}}(y) \mathrm{d} y$.

The periodogram is always positive so we can integrate over positive values only.

$p_{\gamma_{j}}(z)=\frac{1}{2 S_{j} \mathcal{P}_{j} \sqrt{2 \pi}} \int_{0}^{+\infty} \exp \left\{-\frac{(\ln [y]-M)^{2}}{2 S_{j}^{2}}-\frac{z y}{2 \mathcal{P}_{j}}\right\} \mathrm{d} y$

The dummy variable $w=y / P_{j}$ (and $d y=P_{j} d w$ ) can be used to simplify the above equation:

$p_{\gamma_{j}}(z)=\frac{1}{S_{j} \sqrt{8 \pi}} \int_{0}^{+\infty} \exp \left\{-\frac{\ln [w]^{2}}{2 S_{j}^{2}}-\frac{z w}{2}\right\} \mathrm{d} w$. 
It is worth noting that this formula contains no dependence on the actual value of $\mathcal{P}_{j}$ (and hence $M_{j}$ ). This should not be surprising because we are dealing with the distribution of the ratio of the data to the model, not the absolute value of the data, and so the absolute value of the model is not relevant. The important parameter is $S_{j}$, describing the uncertainty on the model, and this can be calculated from the theory of linear regression (Sect. 3.2). Unlike the ideal case discussed above (Sect. 4.1) the PDF is frequency-dependent, this is reflected in the changes of $S_{j}$ with frequency. As $S_{j} \rightarrow 0$ this formula reduces to the equivalent for the ideal case discussed above.

For a given frequency $f_{j}$ the integral in Eq. (21) can be evaluated numerically to give the PDF for $\hat{\gamma}_{j}$. Figure $7 \mathrm{com}$ pares the prediction of Eq. (21) with a Monte Carlo distribution at two different frequencies. Also shown for comparison is the $\chi_{2}^{2}$ PDF which represents the distribution in the absence of uncertainties on the model (i.e. $S_{j} \rightarrow 0$ ). At small $\hat{\gamma}$ (i.e. low significance peaks) the two distributions agree, whereas for large $\hat{\gamma}$ (i.e. high significance peaks) there is a substantial difference between the PDFs including and excluding the uncertainty on the model. This means that, while the effect of including the uncertainty on the model is negligible for low significance peaks, the significance of high significance peaks may be substantially overestimated if this additional uncertainty is not taken into account.

The probability of obtaining a value of $\hat{\gamma}_{j}$ higher than $\gamma_{\epsilon}$ can be computed by integrating this PDF:

$\operatorname{Pr}\left\{\hat{\gamma}_{j}>\gamma_{\epsilon}\right\}=\int_{\gamma_{\epsilon}}^{\infty} p_{\gamma_{j}}(z) \mathrm{d} z=\epsilon_{1}$.

This can be evaluated numerically to find $\gamma_{\epsilon}$ for a given $\epsilon_{1}$. Equivalently, we can find the value of $\gamma_{\epsilon}$ at the corresponding $1-\epsilon_{1}$ significance level:

$\operatorname{Pr}\left\{\hat{\gamma}_{j}<\gamma_{\epsilon}\right\}=\int_{0}^{\gamma_{\epsilon}} p_{\gamma_{j}}(z) \mathrm{d} z=1-\epsilon_{1}$.

The calculation of $\gamma_{\epsilon}$ depends only on $p_{\gamma_{j}}(z)$, from Eq. (21), which in turn depends only on $S_{j}$, from Eq. (13), and this is calculated using the the abscissae (frequencies $f_{j}$ ) with no dependence on the ordinates (periodogram powers $I_{j}$ ). The critical value $\gamma_{\epsilon}$ can be evaluated using only the frequencies of the periodogram.

Finally, we need to correct for the number of frequencies examined. The probability that a peak will be seen given that $n^{\prime}$ frequencies were examined is $\epsilon_{n^{\prime}}=1-\left(1-\epsilon_{1}\right)^{n^{\prime}}$. One can find the global $\left(1-\epsilon_{n^{\prime}}\right) 100$ per cent confidence level by finding the value $\gamma_{\epsilon}$ that satisfies:

$\int_{\gamma_{\epsilon}}^{\infty} p_{\gamma_{j}}(z) \mathrm{d} z=1-\left(1-\epsilon_{n^{\prime}}\right)^{1 / n^{\prime}} \approx \epsilon_{n^{\prime}} / n^{\prime}$

where $n^{\prime}$ is again the number of frequencies examined.

As an illustration of the effect of the model uncertainty, consider a peak in the $j=10$ frequency bin of a $n=128$ periodogram. Neglecting the effect of model uncertainty the nominal $\epsilon_{1}=10^{-4}$ threshold is $\gamma_{\epsilon}=18.42$ (using Eq. (15)). But, after including the uncertainty in the model, the probability of this level being exceeded is really $3.6 \times 10^{-4}$ (using Eq. (22)).

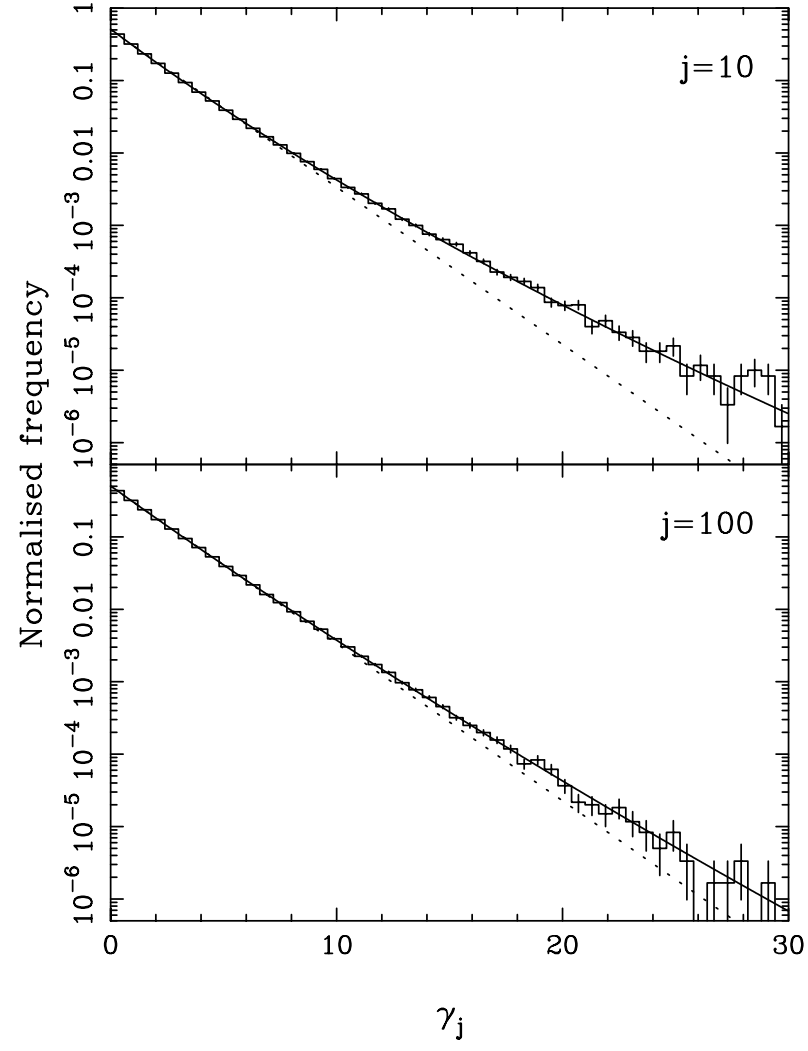

Fig. 7. Monte Carlo demonstration of the PDF of the ratio $\hat{\gamma}_{j}$. The histograms represent the distribution of the ratio $\hat{\gamma}_{j}$ (measured at the $j=10$ and $j=100$ Fourier frequencies) from $10^{6}$ Monte Carlo realisations of $K=256$ time series (with an $\alpha=2, N=1$ spectrum). For each of the $10^{6}$ simulated time series the periodogram was fitted using the LS method, after ignoring frequency $j$, and the ratio $\hat{\gamma}_{j}=2 I_{j} / \hat{\mathcal{P}}_{j}$ was measured. The solid curve marks the predicted PDF using Eq. (21) and the dotted line marks the PDF of a $\chi_{2}^{2}$ distribution (Eq. (3)). The difference between the two model PDFs is due to the uncertainty on the model fit. (Compare with Fig. 5 of Israel \& Stella 1996).

For $n=128$ trials this corresponds to global significances of 98.7 per cent confidence (ignoring the model uncertainty) and 95.4 per cent confidence (including the model uncertainty). The first of these might be called a significant detection, but once the model uncertainty is taken into account the detection is no longer very significant. The difference is even more profound for higher significances.

\section{Verification of the method}

The procedures discussed above were tested using Monte Carlo simulations. The simulations measured the type I error rate, or the rate of "false positive" (spurious) detections of periodic signals. For this experiment many artificial time series were generated based on a power law spectrum (i.e. the null hypothesis). For each simulation the $\gamma_{\epsilon}$ threshold, corresponding to a $1-\epsilon$ confidence level, was calculated and the number of periodogram ordinates that exceeded this value were recorded. The rate measured from the Monte Carlo simulations should be the same as the false alarm probability $\epsilon$, often called the "size of the test", which is the expected rate of type I errors. If the 

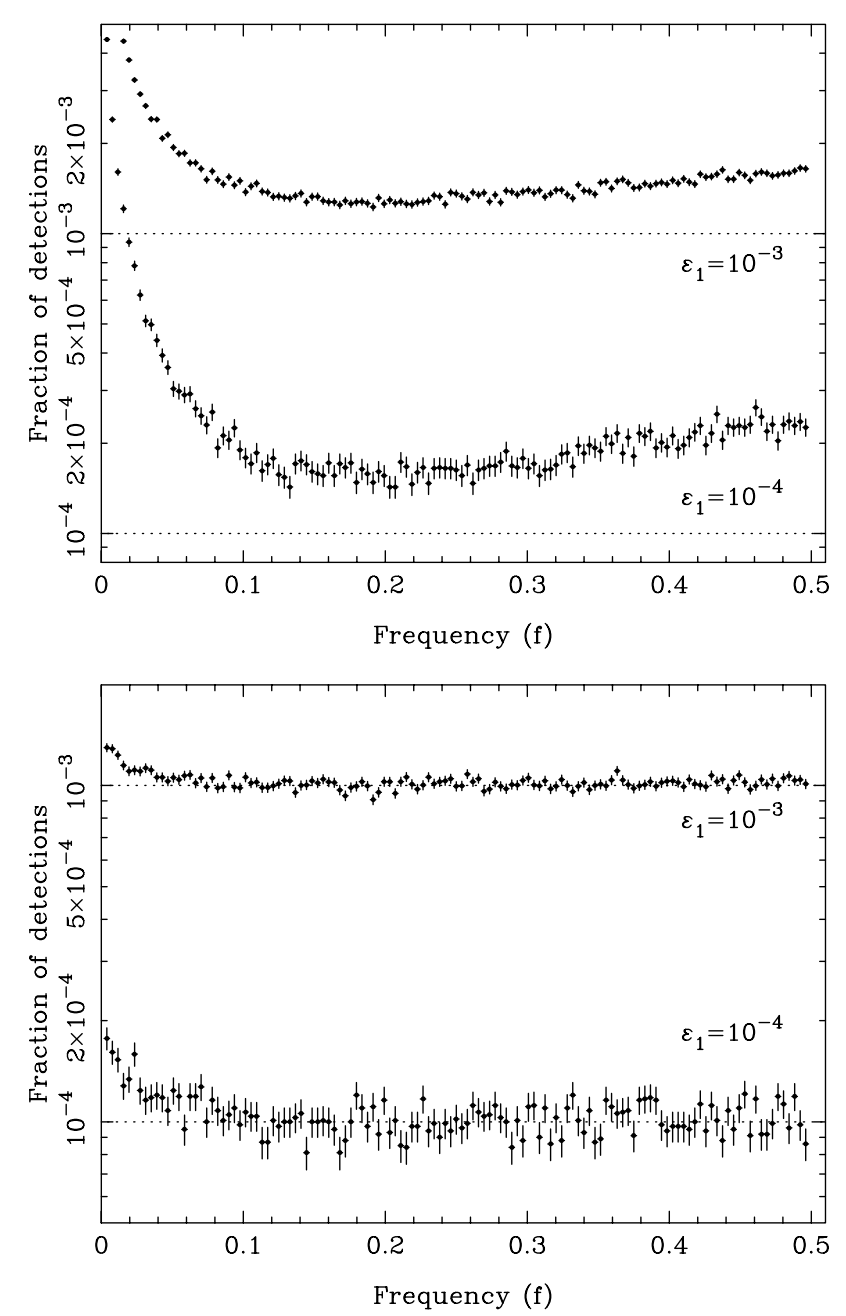

Fig. 8. Monte Carlo study of the performance of tests for significant periodogram peaks. For each panel $10^{6}$ random time series of length $K=256$ were generated (with an $\alpha=2, N=1$ spectrum). For each of the 127 frequencies tested $(=K / 2-1$ since the Nyquist frequency was ignored) the fraction of Monte Carlo simulations with a peak exceeding the nominal 99.9 and 99.99 per cent confidence levels was recorded. The upper panel used the confidence levels from Eq. (15). The lower panel used the confidence levels computed with Eq. (22) which accounts for the uncertainty in the model. The error bars were computed using $\sqrt{p(1-p) / N}$ where $N$ is the number of simulations.

observed rate of false detections exceeds the nominal size of the test then one should expect an excess of spurious detections (detections may not be reliable). If the observed rate falls below the nominal test size then the test is conservative (it gives even fewer spurious detections than expected).

The Monte Carlo rate was derived from $10^{6}$ random time series of length $K=256$ (generated with a $\alpha=2, N=1$ spectrum). For each series the periodogram was computed and fitted using the LS method. In the first run, the effects of the uncertainty on the model were ignored and the $\gamma_{\epsilon}$ thresholds were computed for $\epsilon_{1}=10^{-3}$ and $10^{-4}$ using Eq. (15). These corresponds to 99.9 and 99.99 per cent confidence levels in a single trial test. For each frequency the fraction of simulations that show peaks larger than the threshold was recorded. As shown in Fig. 8 (upper panel) the observed rate of type I errors in the

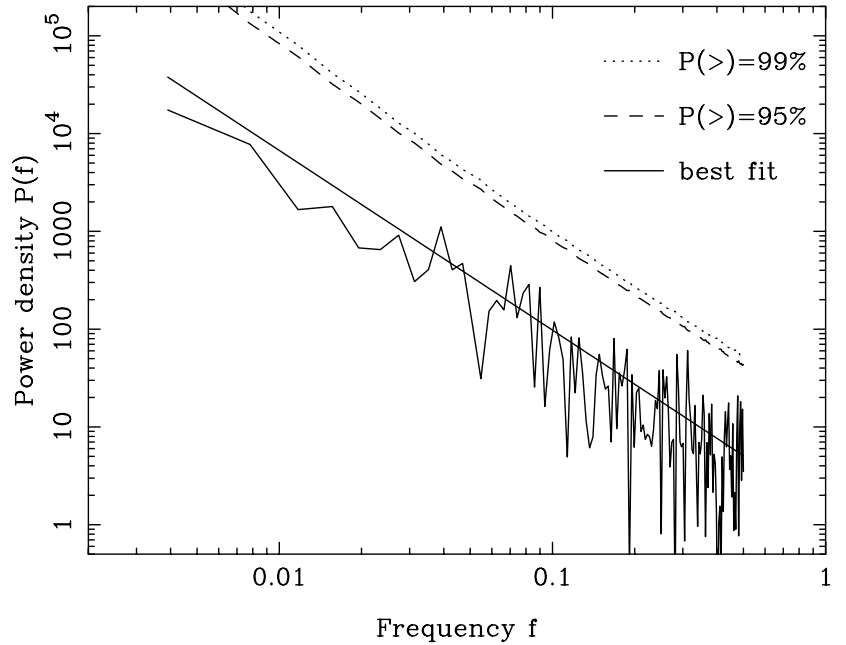

Fig. 9. Same periodogram as in Fig. 1. Plotted are the de-biased LS estimate of the power law spectral model (solid curve) and the 95 and 99 per cent upper limits (dotted curves) on the expected power (global significance levels for $n=127$ independent frequencies).

simulated data was far in excess of the nominal size of the test. Thus the actual rate of spurious detections was higher than the nominal test size, and greatly so at low frequencies where the model is more uncertain. The situation is worse at high significances (small test sizes) because the tail of the PDF diverges from the expectation (Fig. 7). This means that significances calculated by Eq. (15) will be overestimated.

The situation is much better when the $\gamma_{\epsilon}$ threshold was computed (again for $\epsilon_{1}=10^{-3}, 10^{-4}$ ) using Eq. (22). Again $10^{6}$ random time series were generated with $K=256$ using the same spectrum. For each periodogram the model power $\hat{\mathcal{P}}_{j}$, and its error parameters $S_{j}$, were computed by ignoring frequency $f_{j}$ and then fitting with the LS method. The ratio $\hat{\gamma}_{j}$ was compared to the critical threshold $\gamma_{\epsilon}$ (computed from Eq. (22)) at each frequency. The fraction of Monte Carlo simulations that showed "significant" peaks was very close to the expected level (the nominal size) and independent of frequency. The exceptions are the $\approx 5$ lowest frequencies. Here the model is least constrained and the assumption implicit in Eq. (11), that the distribution of $\log \left[\hat{\mathcal{P}}_{j}\right]$ is normal, becomes inaccurate. For $j \gtrsim 5$ the confidence levels predicted by Eq. (22) gave the correct rate of type I errors.

Figure 9 shows a specific example, namely the same data as in Fig. 1, with the LS power law model. Also shown are the ("global") $n^{\prime}$-trial confidence limits computed as discussed in Sect. 4.2. Clearly none of the peaks in the periodogram exceeds the 95 per cent limit, as expected for red noise.

\section{Caveats and comparison with other methods}

\subsection{Underlying assumptions}

In order for the test to give reliable significance limits the underlying noise spectrum must be a power law. Clearly if the broad-band noise spectrum does not resemble a power law the results of the LS fitting will not be valid. The general solution to this problem is to replace the LS fitting procedure with the 
exact maximum likelihood (ML) procedure for fitting the $\chi_{2}^{2}$ distributed periodogram. The appendix describes this method.

The test was intended to be used for assessing the significance of peaks in the periodograms of X-ray observations of Seyfert galaxies, which tend to be rather short $K \sim 10^{3}$ and also show significant variance due to measurement errors (Poisson noise). These measurement uncertainties produce a flat component that is added to the source power spectrum in the periodogram. The effect will cause the observed spectrum to flatten at high frequencies as the power in the red noise spectrum of the source becomes comparable to the power in the flat Poisson noise spectrum. Using the normalisation given in Eq. (1) the expected Poisson noise level is $P_{N}=2(\langle x\rangle+B) /\langle x\rangle^{2}$ where $\langle x\rangle$ is the mean count rate and $B$ is the mean background rate ${ }^{3}$. It is also now known that at low frequencies the power spectra of Seyferts break from a single power law (e.g. Uttley et al. 2002; Markowitz et al. 2003). These deviations from a single power law should be accounted for in modelling the spectrum. The simplest solution is to divide the periodogram into frequency ranges within which the power spectrum is approximately a single power law. The period detection test can then be used as described above. The crucial point is that as long as the periodogram can be fitted reasonably well with a power law over a frequency range of interest (as judged using the KS test) the test will be valid. Alternatively one may fit a model of a power law plus constant (to account for the flattening) using the ML method discussed in the Appendix.

Furthermore, the test, which is based on the discrete Fourier transform, is most sensitive to sinusoidal periodicities. Non-sinusoidal variations will have their power spread over several frequencies which will lessen the detection significance in any one given frequency. Other methods such as epoch folding (Leahy et al. 1983), whereby one bins the time series into phase bins at a test period, can be more sensitive to such variations. At the correct period the periodic variations will sum while any background noise will cancel out, thus revealing the profile of the periodic pulsations. However, the background noise will only cancel out if it is temporally independent, i.e. white noise. Again, the presence of any underlying red noise variations may produce unreliable results (Benlloch et al. 2001) if not correctly accounted.

\subsection{Comparison with other methods}

\subsubsection{Lomb-Scargle periodogram}

The method described above is based around the standard (Fourier) periodogram and therefore requires uniformly sampled time series. This ensures the asymptotic independence of the periodogram ordinates. If the time series is non-uniformly sampled one may use other periodogram estimators such as the Lomb-Scargle periodogram (Lomb 1976; Scargle 1982; Press \& Rybicki 1989). However, the behaviour of these will not be identical to that discussed above. The above procedure should

\footnotetext{
${ }^{3}$ For the case of Gaussian errors $\sigma_{k}$ on each flux measurement the expected Gaussian noise level is $P_{N}=2 \Delta T\left\langle\sigma^{2}\right\rangle /\langle x\rangle^{2}$. This assumes the time series comprises contiguous bins of length $\Delta T$.
}

not be used on non-uniformly sampled time series (nor should the method discussed in Sect. 13.8 of Press et al. 1996 be used in the presence of non-white noise). Zhou \& Sornette (2001) discuss the results of Monte Carlo tests on the distribution of peaks in the Lomb-Scargle periodogram for various types of processes.

\subsubsection{Oversampled periodogram}

Oversampling the periodogram, i.e. calculating periodogram ordinates at frequencies between the normal Fourier frequencies, is sometimes done in order to increase the sensitivity to weak signals that lie at frequencies between two Fourier frequencies. A periodic variation with a frequency nearly equidistant between two adjacent Fourier frequencies, e.g. between $f_{j}$ and $f_{j+1}$, will have its power spread (almost entirely) between these two frequencies, thus reducing the significance in any one frequency bin. The reduction in power per bin can be as much as $\approx 4 / \pi^{2} \approx 0.41$. In these situations oversampling the periodogram by including additional frequencies between $f_{j}$ and $f_{j+1}$ can increase sensitivity to the periodicity. However, it must also be noted that by oversampling the periodogram one is testing more than $K / 2$ frequencies, allowing many more opportunities to find spurious peaks. The number of trials increases above the usual $n=K / 2$ case if the periodogram is oversampled, although the effective number of independent trials does not scale linearly with the oversampling factor because there is a fixed number $(n)$ of strictly independent frequencies (the Fourier frequencies).

Oversampling the periodogram and assuming $\approx n$ trials will tend to overestimate the significance of peaks in the periodogram. This was demonstrated by Monte Carlo simulations (see Fig. 10). For this demonstration $10^{4}$ white noise time series (spectrum: $\alpha=0, N=1$ ) were generated with length $K=256$ and for each one the periodogram was calculated using both the standard Fourier frequencies and also oversampling the frequency resolution by a factor 8 . The peak power in the periodogram of each of the simulated time series was recorded. The distribution of peak powers is shown in Fig. 10 for both the standard and the oversampled periodograms. There is an obvious tendency for the oversampled periodogram to show slightly higher peak values, as might be expected based on the above arguments. These peak powers were translated to "global" significances (assuming $n$ independent trials) and the distribution of significances is shown in the lower panel of Fig. 10. The distribution is flat for the Fourier sampled periodogram: the significance of the peaks is exactly as expected. The distribution derived from the oversampled periodogram clearly shows a substantial excess of high significance peaks. This means that the oversampled periodogram is likely to produce many more spurious peaks than expected if one assumes only $n$ independent trials were made. A Monte Carlo estimate of the global significance would be required to calibrate the significance of peaks in oversampled periodogram (and find a more realistic effective number of trials).

An alternative to oversampling the periodogram is to perform a sliding two-bin search for periodogram peaks 

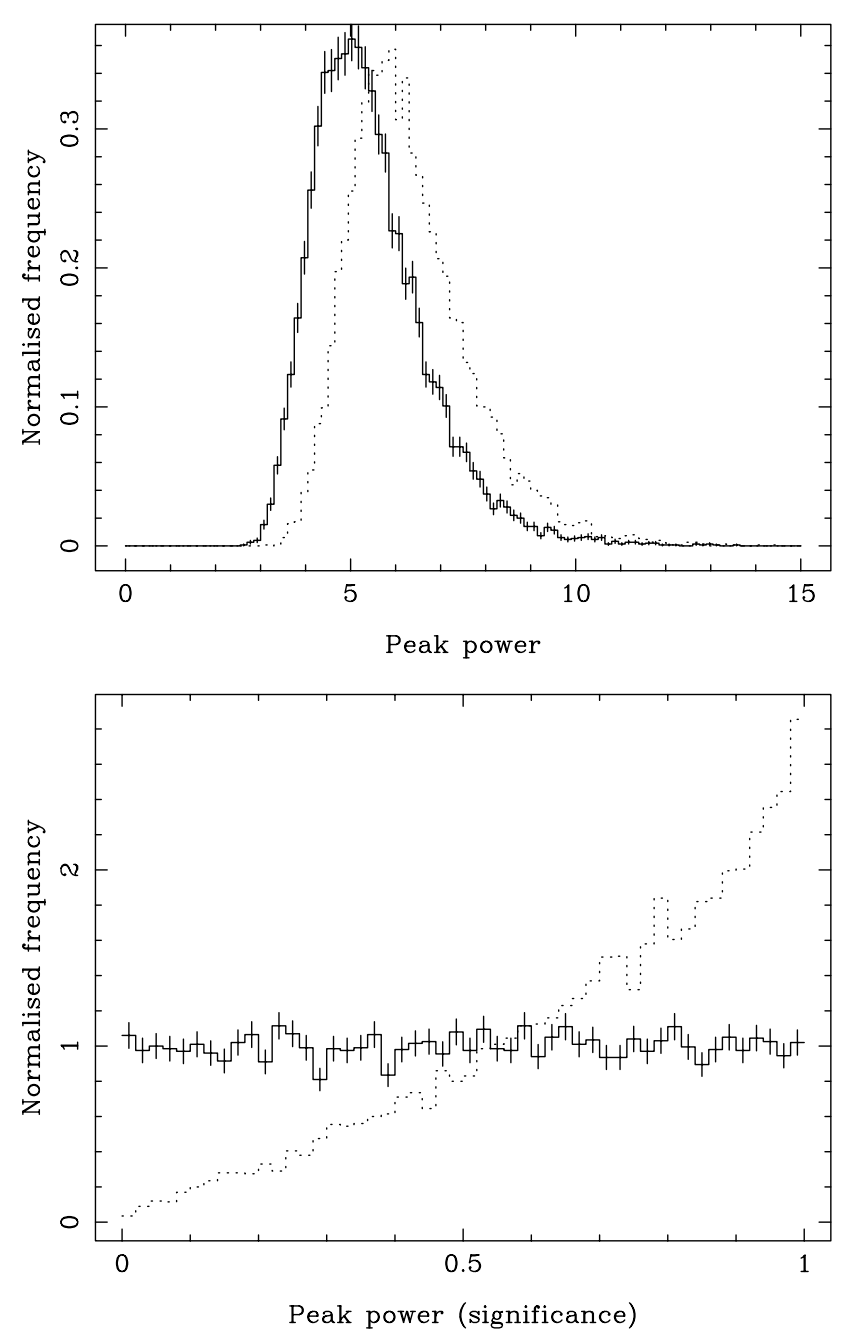

Fig. 10. Comparison between periodograms sampled at the $K / 2$ Fourier frequencies and oversampled by a factor 8 . The upper panel shows the distribution of peaks in the periodogram from $10^{4}$ random white noise time series of length $K=256$ (using an $\alpha=0, N=1$ spectrum). For each simulation the value of the peak of the periodogram was recorded for the standard (solid histogram) and oversampled (dotted histogram) case. The oversampled periodograms clearly show slightly larger peak values. The lower panel shows the same data except the peak powers have been converted to "global" significances using Eq. (16).

using the standard Fourier frequencies only, as discussed by van der Klis (1989; Sect. 6.4). This increases the sensitivity to periods whose frequencies fall between the Fourier frequencies. However, one will still need to perform Monte Carlo simulations to assess the global significance (by measuring the rate of type I errors in the simulations).

\subsubsection{Monte Carlo testing}

An alternative test for periodic variations in red noise is to estimate the likelihood of observing a given peak using Monte Carlo simulations of red noise processes (Benlloch et al. 2001; Halpern et al. 2003). However, the method of Benlloch et al. (2001) does not account for uncertainties in the best-fitting model, which can seriously effect the apparent significances of strong peaks (Sect. 4.2). Monte Carlo simulations are only as good as the model they assume! One solution would be to map the (multi-dimensional) distribution of the model parameters and for each simulation draw the model parameters at random from this distribution. This would thereby account for the uncertainty in the model parameters. (Protassov et al. 2002 use a similar approach to calculate posterior predictive $p$-values.)

\section{Discussion}

A simple procedure is presented for assessing the significance of peaks in a periodogram when the underlying continuum noise has a power law spectrum.

\subsection{Recipe}

The following is one possible recipe for periodogram analysis.

- Calculate the periodogram of the data $I\left(f_{j}\right)$ (Sect. 2) and convert to $\log$-space, i.e. $\log \left[f_{j}\right]$ and $\log \left[I\left(f_{j}\right)\right]$.

- Ignore the Nyquist frequency and frequencies above which Poisson noise is significant, leaving $n^{\prime}$ frequencies.

- Estimate the power law parameters by fitting a linear function to the log-periodogram using the LS method (Sect. 3).

- Test the goodness of the fit by comparing the distribution of data/model residuals with the $\chi_{2}^{2}$ expectation using a KS test (Sect. 3).

- Calculate the "global" ( $n^{\prime}$-trial) threshold ratio for a (1 $\epsilon)$ significance detection using $\gamma_{\epsilon}=-2 \ln \left[1-(1-\epsilon)^{1 / n^{\prime}}\right]$ where $n^{\prime}$ is number of periodogram points used in the fit and $\epsilon$ is the desired "false alarm probability" (Sect. 4.1).

- Multiply the best-fitting continuum model by $\gamma_{\epsilon} / 2$ (or add its logarithm in log-space).

This will provide an estimate of the power spectral slope and normalisation and a first indication of the presence of significant periodicities. However, as discussed in Sect. 4.2 the uncertainty inherent in the model fitting means that the significances of strong peaks may be substantially overestimated. This procedure should be quite accurate for low significance peaks however, meaning that the above procedure is valid for rejecting low significance peaks $\left(\epsilon_{1}>0.01\right)$. The inaccuracy of the method at high powers means that the procedure should only be used to reject low significance peaks, not detect high significance peaks. In order to obtain a more rigorous estimate of the significance of stronger peaks one needs to account for the additional uncertainty in the model.

For a given frequency of interest, $f_{j}$, one must:

- Ignore $f_{j}$ from the fit (in order that the data and model are independent at $f_{j}$ ).

- Re-fit the periodogram using the LS method (Sect. 3).

- Calculate the power in the model at this frequency, $\hat{\mathcal{P}}_{j}$.

- Measure the ratio $\hat{\gamma}_{j}=2 I_{j} / \hat{\mathcal{P}}_{j}$.

- Compute the uncertainties on the model parameters (Sect. 3.2) and hence the uncertainty on the model continuum $S_{j}$ (Sect. 3.3). 
- Calculate the probability of observing a value of $\hat{\gamma}_{j}$ this high by numerically integrating Eq. (22).

The above procedure is essentially the application of two standard tools for time series analysis. The first is estimating the power law spectral properties by LS fitting of the log-periodogram. This has been discussed by several authors (e.g. Geweke \& Porter-Hudak 1983; Fougere 1985; Pilgram \& Kaplan 1998). The second tool is applying the known $\chi_{2}^{2}$ properties of the periodogram to estimate confidence levels, as is standard for white noise spectra (Priestley 1981; van der Klis 1989). The additional calculation to include the uncertainty in the model follows standard statistical procedures. The overall approach is similar to that discussed by Israel \& Stella (1996) but is tailored to power law spectra.

In order for the method to produce reliable results the underlying power spectrum has to be a power law. However, the test will work well even for data that show deviations from a power law (such as intrinsic low frequency flattening or high frequency flattening due to Poisson noise) provided the periodogram is divided into frequency intervals within which a single power law provides a good description of the data (determined with a KS test). In this case the power spectrum over the restricted frequency ranges is indistinguishable from a power law and, when applied to these limited frequency ranges, the method will function as expected.

\subsection{Application to real data}

As a demonstration of the method we present a re-analysis of the X-ray observations of two Seyfert galaxies. The first is the long ASCA observation of IRAS 18325-5926 (Iwasawa et al. 1998) and the second is the XMM-Newton GTO observation of Mrk 766 (Boller et al. 2001). For IRAS 18325-5926 a background-subtracted $0.5-10 \mathrm{keV}$ SIS0 light curve was extracted in 100-s bins, using a 4 arcmin radius source region, and rebinned onto an evenly spaced grid at the spacecraft orbital period (5760-s). For Mrk 766 a background-subtracted, exposure-corrected $0.2-2 \mathrm{keV}$ light curve was extracted from the EPIC pn using a 38 arcsec radius source region. The rebinned IRAS 18325-5926 light curve contained 84 regularly spaced bins and the Mrk 766 light curve contained 330 contiguous 100-s bins.

The periodogram of each light curve was calculated and the expected Poisson noise level determined. Only the lowest frequency periodogram points were examined, to minimise the effect of the Poisson noise level. For IRAS 18325-5926 only the 19 lowest frequency points were used, while for Mrk 766 only 97 points were used. These were fitted with a linear function in log-space, giving slopes of $\alpha \approx 0.9$ and $\alpha \approx 1.8$ for IRAS 18325-5926 and Mrk 766, respectively. The 95 and 99 per cent confidence limits on the periodogram were computed, accounting for the number of frequencies examined in each case (see Figs. 11 and 12). In neither object did a single periodogram point exceed the 95 per cent limit, meaning that there is no strong evidence to suggest a periodic component to their variability, contrary to the original claims of Iwasawa et al. $\left(1998 ; f_{\mathrm{P}} \approx 1.7 \times 10^{-5} \mathrm{~Hz}\right)$ and Boller et al. (2001;

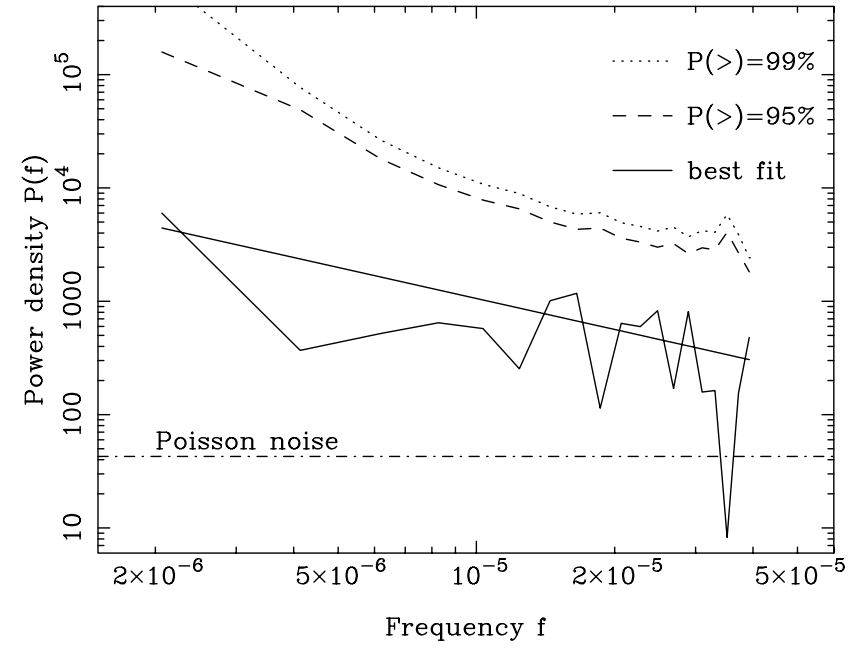

Fig. 11. Periodogram of IRAS 18325-5926 from ASCA. Plotted are the de-biased LS estimate of the power law spectral model (solid curve) and the 95 and 99 per cent upper limits (dotted curves) on the expected power (global significance levels for $n=19$ independent frequencies). Also shown is the expected level of the Poisson noise power.

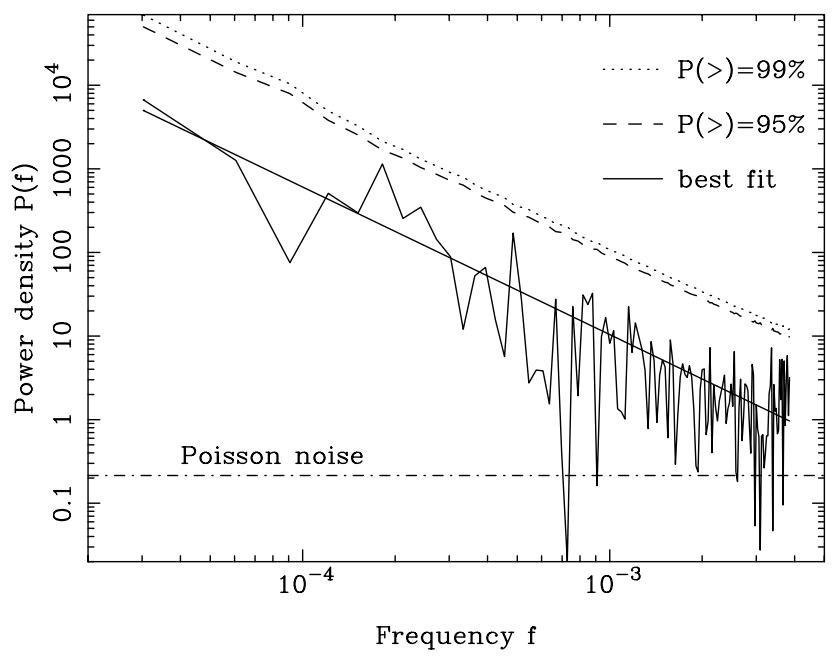

Fig. 12. Periodogram of Mrk 766 from XMM-Newton.

$f_{\mathrm{P}} \approx 2.4 \times 10^{-4} \mathrm{~Hz}$ ). Benlloch et al. (2001) drew similar conclusions based on Monte Carlo simulations.

\subsection{Conclusions}

The simplicity of the proposed test means that it can be used as a quick, first check against spurious periodogram peaks, without the need for extensive Monte Carlo simulations. Examples where this test might be useful include not only X-ray observations of Seyferts but also testing for periodic components in monitoring of blazars (e.g. Kranich et al. 1999; Hayashida et al. 1998), the Galactic centre (Genzel et al. 2003) and other astrophysical sources that show red noise variations.

The basic idea behind the method is to de-redden the periodogram by dividing out the best-fitting power law and then using the known distribution of the periodogram ordinates to estimate the likelihood of observing a given peak if the null 
hypothesis (power law spectrum with no periodicity) is true. However, this should not be treated as a "black box" solution to the problem of detecting periodicities in red noise data. There is no substitute for a thorough understanding of the nuances of power spectral statistics, as illustrated by the PDFs discussed in Sect. 4.2. The tail of the PDF is very sensitive to the details of the method, and thus one needs to treat any periodogram analysis with great care in order to avoid overestimating the significance of a peak.

To date the most significant candidate periodicities found in the X-ray variations active galaxies are those in long $E U V E$ observations of three nearby Seyferts by Halpern et al. (2003). However, their published global significances will be overestimates for two reasons: $(i)$ no account was made of the uncertainty on the model and (ii) the periodograms were oversampled. These two separate issues both act to artificially boost the apparent significance of spurious signals, as discussed in Sects. 4.2 and 6.2.2, respectively. Similarly the apparently significant candidate periodicity in NGC 5548 found by Papadakis \& Lawrence (1993b) was shown to be substantially less significant when the uncertainty in the modelling was included (Tagliaferri et al. 1996). This leaves us in the interesting situation of there being not one surviving, robustly determined and significant ( $>99$ per cent) periodicity in the X-ray variability of an active galaxy.

Finally we close with a plea. There exist many more AGN light curves and periodograms than have been published. There is a natural publication bias: those that show the most "significant" features get published. This means the true number of trials is much larger than for any one given experiment. In such situations one should treat with caution the significance of the subset of results that are published (e.g. Scargle 2000). That is, until a result is published that is so significant that it cannot be accounted for by publication bias. The detection of periodic or quasi-periodic variations in galactic nuclei would be a major discovery and of great importance to the field. The importance of the result should be argument enough for very high standards of discovery. We would therefore advocate serious further investigation of only those candidate periodicities with high significances ( such as a $>99.9$ per cent confidence, or a " $3 \sigma$ minimum," criterion) after accounting for all likely sources of error.

Acknowledgements. I acknowledge financial support from the PPARC, UK. I also thank Phil Uttley for many useful discussions and Iossif Papadakis for a very thorough referee's report that encouraged me to expand several sections of the paper.

\section{Appendix A: Maximum likelihood (ML) fitting}

In this section we briefly elucidate the exact method of maximum likelihood (ML) fitting periodograms. See Anderson, Duvall \& Jefferies (1990) and Stella et al. (1997) for more details. The probability density function (PDF) of the periodogram ordinates at each frequency are given by:

$p\left(I_{j}\right)=\frac{1}{\mathcal{P}_{j}} \mathrm{e}^{-I_{j} / \mathcal{P}_{j}}$ where $\mathcal{P}_{j}$ is the true underlying spectrum (the expectation value of the periodogram) at frequency $f_{j}$. This is valid for frequencies $j=1,2, \ldots, n-1$ since the periodogram ordinate at the $j=n$ (Nyquist) frequency is distributed differently.

Assuming a model $\hat{\mathcal{P}}_{j}\left(\hat{\theta}_{k}\right)$, determined by parameters $\hat{\theta}_{k}=$ $\left\{\hat{\theta}_{1}, \hat{\theta}_{2}, \ldots, \hat{\theta}_{M}\right\}$, we can write the joint probability density of observing the $n-1$ periodogram ordinates:

$\mathcal{L}=\prod_{j=1}^{n-1} p\left(I_{j}\right)=\prod_{j=1}^{n-1} \frac{1}{\hat{\mathcal{P}}_{j}} \mathrm{e}^{-I_{j} / \hat{\mathcal{P}}_{j}}$.

If the data $I_{j}$ have already been observed this represents the likelihood function. Maximising the likelihood $\mathcal{L}$ is equivalent to minimising $S \equiv-2 \ln [\mathcal{L}]$ and we can rewrite this as:

$S=2 \sum_{j=1}^{n-1}\left\{\ln \left[\hat{\mathcal{P}}_{j}\right]+\frac{I_{j}}{\hat{\mathcal{P}}_{j}}\right\}$.

Finding the model parameters $\hat{\theta}_{k}$ that minimise $S$ will yield the maximum likelihood parameter values.

One can then use standard tools of maximum likelihood analysis, such as the likelihood ratio test (LRT) to test for additional free parameters in the model:

$R=-2 \ln \left[\mathcal{L}_{1} / \mathcal{L}_{2}\right]=S_{1}-S_{2}$

where $\mathcal{L}_{1}$ represents the likelihood for the simpler (more parsimonious) model and $\mathcal{L}_{2}$ represents the likelihood for the model with the additional free parameters. The second, more complex model, always contains within it the simpler model as a subset (the models are nested) and therefore the likelihood for the more complex model is always equal to or greater than that for the simpler model. With certain restrictions (see Protassov et al. 2002) $R$ is distributed as a $\chi_{v}^{2}$ variable where $v$ is the number of additional free parameters. This test could be used, for example, to compare nested models such as broken and unbroken power laws.

Alternatively one can compare different models using the Akaike Information Criterion (AIC; Akaike 1973):

$A I C_{i}=-2 \ln \left[\mathcal{L}_{i}\right]+2 k_{i}=S_{i}+2 k_{i}$

where $\mathcal{L}_{i}$ is the likelihood and $k_{i}$ is the number of free parameters for model $i$. The second term in the sum is a penalty for including more free parameters. The model that minimises the $A I C$ is considered to be the best (the models need not be nested).

One can also use $\Delta S=-2 \Delta \ln [\mathcal{L}]$ to place confidence limits on the model parameters in a fashion exactly analogous to mapping confidence contours using $\Delta \chi^{2}$ (Sect. 15.6 of Press et al. 1996). Under fairly general conditions (see Cash 1979), e.g. the $\ln [\mathcal{L}]$-surface is approximately shaped like a multi-dimensional paraboloid, $\Delta S$ is distributed as $\chi_{v}^{2}$ where $v$ is the number of parameters of interest (e.g. $v=1$ for the one-dimensional confidence region on an individual parameter). One can use standard tables of $\chi_{v}^{2}$ values to place confidence limits (e.g. $\Delta S=2.71$ corresponds to 90 per cent confidence limits on one parameter). 
For the purposes of period searching one may fit a suitable $M$-parameter continuum model (representing the null hypothesis, i.e., no periodic signal) using the ML method and define the $M$-dimensional distribution of its parameters (using $\Delta S$ ). One can then use this $M$-dimensional distribution of the model parameters to randomly draw models for Monte Carlo simulation. This procedure will thereby account for the likely distribution of model parameters (which gives rise to uncertainties in the estimated continuum level).

\section{References}

Abramowicz, M. A., Kluzniak, W., McClintock, J. E., \& Remillard, R. A. 2004, ApJ, 609, L63

Abramowitz, M., \& Stegun, I. A. 1964, Handbook of Mathematical Functions, Applied Mathematics Series, Vol. 55 (New York: Dover Publications)

Akaike, H. 1973, in 2nd International Symposium on Information Theory, ed. B. N. Petrov, \& F. Csáki (Budapest: Akadémiai Kiado)

Anderson, E. R., Duvall, T. L., \& Jefferies, S. M. 1990, ApJ, 364, 699

Benlloch, S., Wilms, J., Edelson, R., Yaqoob, T., \& Staubert, R. 2001, ApJ, 562, L121

Bevington, P. R., \& Robinson, D. K. 1992, Data Reduction and Error Analysis for the Physical Sciences (New York: McGraw-Hill)

Bloomfield, P. 2000, Fourier Analysis of Time Series (New York: Wiley)

Boller, Th., Keil, R., Trümper, J., et al. 2001, A\&A, 365, L146

Cash, W. 1979, ApJ, 228, 939

Chatfield, C. 1989, The analysis of time series: theory and practice, 4th ed. (London: Chapman \& Hall)

Fisher, R. A. 1929, Proceedings of the Royal Society on London: Series A, 125, 54

Fougere, P. F. 1985, J. Geophys. Res., 90, 4355

Genzel, R., Schödel, R., Eckart, A., et al. 2003, Nature, 425, 934

Geweke, J., \& Porter-Hudak, S. 1983, Journal of Time Series Analysis, 4, 221

Groth, E. J. 1975, ApJS, 29, 285

Halpern, J. P., Leighly, K. M., \& Marshall, H. L. 2003, ApJ, 585, 665 Hayashida, N., Hirasawa, H., Ishikawa, F., et al. 1998, ApJ, 504, L71 Israel, G. L., \& Stella, L. 1996, ApJ, 468, 369

Iwasawa, K., Fabian, A. C., Brandt, W. N., et al. 1998, MNRAS, 295, L20

Jenkins, G. M., \& Watts, D. G. 1968, Spectral Analysis and its Applications (San Fancisco: Holden-Day)
Koen, C. 1990, ApJ, 348, 700

Kranich, D., deJager, O. C., Kestel, M., et al. 1999, in Proc. of 26th International Cosmic Ray Conference (Salt Lake City), 3, 358

Lawrence, A., Watson, M. G., Pounds, K. A., \& Elvis, M. 1987, Nature, 325, 694

Leahy, D. A., Darbro, W., Elsner, R. F., et al. 1983, ApJ, 266, 160

Lomb, N. R. 1976, Ap\&SS, 39, 447

Markowitz, A., Edelson, R., Vaughan, S., et al. 2003, ApJ, 593, 96

$\mathrm{M}^{\mathrm{c} C l i n t o c k, ~ J . ~ E ., ~ \& ~ R e m i l l a r d, ~ R . ~ A . ~ 2004, ~ i n ~ C o m p a c t ~ S t e l l a r ~ X-r a y ~}$ Sources, ed. W. H. G. Lewin, \& M. van der Klis (Cambridge: Cambridge University Press), in press [arXiv:astro-ph/0306213]

Papadakis, I. E., \& Lawrence, A. 1993a, MNRAS, 261, 612

Papadakis, I. E., \& Lawrence, A. 1993b, Nature, 361, 233

Pilgram, B., \& Kaplan, D. T. 1998, Physica D, 114, 108

Press, W. H. 1978, Comments Astrophys., 7, 103

Press, W. H., \& Rybicki, G. B. 1989, ApJ, 338, 277

Press, W. H., Teukolsky, S. A., Vetterling, W. T., \& Flannery, B. P. 1996, Numerical Recipes (Cambridge: Cambridge Univ. Press)

Priestley, M. B. 1981, Spectral Analysis and Time Series (London: Academic Press)

Protassov, R., van Dyk, D. A., Connors, A., Kashyap, V. L., \& Siemiginowska, A. 2002, ApJ, 571, 545

Scargle, J. D. 1982, ApJ, 263, 835

Scargle, J. D. 2000, Journal of Scientific Exploration, 14, 91 [arXiv:physics/9909033]

Schuster, A. 1898, Terrestrial Magnetism, 3, 24

Stella, L., Arlandi, E., Tagliaferri, G., \& Israel, G. L. 1997, in Applications of Time Series Analysis in Astronomy and Meteorology, ed. T. Subba Rao, M. B. Priestley, \& O. Lessi (London: Chapman \& Hall) [arXiv: astro-ph/9411050]

Tagliaferri, G., Bao, G., Israel, G. L., Stella, L., \& Treves, A. 1996, ApJ, 465, 181

Timmer, J., \& König, M. 1995, A\&A, 300, 707

Uttley, P., McHardy, I. M., \& Papadakis, I. 2002, MNRAS, 332, 231

van der Klis, M. 1989, in Timing Neutron Stars, ed. H. Ogelman, E. P. J. van den Heuvel (Dordrecht: Kluwer), NATO ASI Series C, 262,27

van der Klis, M. 1995, in X-ray Binaries, ed. W. H. G. Lewin, J. van Paradijs, \& E. P. J. van den Heuvel (Cambridge: Cambridge Univ. Press), 252

Wall, J. V., \& Jenkins, C. R. 2003, Practical statistics for astronomers (Cambridge: Cambridge University Press)

Zhou, W.-X., \& Sornette, D. 2001, Int. J. Mod. Phys. C, 13, 137 